\title{
Cyclotron Resonance in Topological Insulators: Non-Relativistic Effects
}

\author{
C. J. Tabert ${ }^{1,2}$ and J. P. Carbotte ${ }^{3,4}$ \\ ${ }^{1}$ Department of Physics, University of Guelph, Guelph, Ontario N1G 2W1 Canada \\ ${ }^{2}$ Guelph-Waterloo Physics Institute, University of Guelph, Guelph, Ontario N1G 2W1 Canada \\ ${ }^{3}$ Department of Physics, McMaster University, Hamilton, Ontario L8S $4 M 1$ Canada and \\ ${ }^{4}$ Canadian Institute for Advanced Research, Toronto, Ontario M5G $1 Z 8$ Canada
}

(Dated: January 1, 2018)

\begin{abstract}
The low-energy Hamiltonian used to describe the dynamics of the helical Dirac fermions on the surface of a topological insulator contains a subdominant non-relativistic (Schrödinger) contribution. This term can have an important effect on some properties while having no effect on others. The Hall plateaus retain the same relativistic quantization as the pure Dirac case. The height of the universal interband background conductivity is unaltered, but its onset is changed. However, the non-relativistic term leads directly to particle-hole asymmetry. It also splits the interband magnetooptical lines into doublets. Here, we find that, while the shape of the semiclassical cyclotron resonance line is unaltered, the cyclotron frequency and its optical spectral weight are changed. There are significant differences in both of these quantities for a fixed value of chemical potential or fixed doping away from charge neutrality depending on whether the Fermi energy lies in the valence or conduction band.
\end{abstract}

PACS numbers: 78.20.Ls, 78.67.-n, 71.70.Di, 72.80.Vp

\section{INTRODUCTION}

Recently, the metallic states that exist at the surface of a topological insulator (TI) have been extensively studied $1-\underline{\underline{-}}$. These display novel physical properties which could possibly be exploited in device applications such as quantum computing ${ }^{8}$. This has lead to a flurry of activity both experimentally and theoretically which has provided new understanding. Many new materials have been discovered $\frac{9,10}{2}$; however, there remains a need to increase the bulk band gap so as to more clearly separate the metallic surface states from the bulk insulating spectrum. The surface states possess an odd number of Dirac points. Here, for simplicity, it is sufficient to consider a single Dirac cone centred at the $\Gamma$ point of the surface Brillouin zone as in $\mathrm{Bi}_{2} \mathrm{Se}_{3}{ }^{11}$. The Hamiltonian which governs the dynamics of the surface fermions involves real $\operatorname{spin}^{12}$ in contrast to graphene and other related materials where instead pseudospin is involved ${ }^{13}-17$. This is due to a strong spin-orbit coupling which provides a dominant linear-in-momentum Dirac contribution to the Hamiltonian with Fermi velocity $v_{F}$. There is also a smaller non-relativistic contribution $\frac{18}{}$, which is quadratic in momentum and characterized by a Schrödinger mass $m$. In this paper, we are particularly interested in understanding the changes in the magneto-optical properties of the helical surface fermions that result from the introduction of the mass term 19 .

The Schrödinger mass term is a prominent feature of the low-energy continuum $\boldsymbol{k} \cdot \boldsymbol{p}$ Hamiltonians ${ }^{9,18}$ which form the basis of our understanding of the TI surface band structure. Indeed, such work was critical in the discovery of three-dimensional (3D) TIs. These simplified Hamiltonians are based on density-functional electronicstructure calculations, as first discussed by Zhang et al $l^{\underline{9}}$ and elaborated upon in Ref $\underset{18}{ }$. For the specific cases of
$\mathrm{Bi}_{2} \mathrm{Te}_{3}$ and $\mathrm{Bi}_{2} \mathrm{Se}_{3}$, the band masses are $m=0.16 m_{e}$ and $0.09 m_{e}$, respectively, where $m_{e}$ is the bare electron mass. Thus, for a magnetic field of 1 Tesla, the respective Schrödinger magnetic energy scales are $\sim 1.25 \mathrm{meV}$ and $0.7 \mathrm{meV}^{\underline{19}}$. The corresponding Dirac energy scale (due to the linear-in-momentum relativistic part of the Hamiltonian) is of order $10 \mathrm{meV}$ (a factor of ten larger than the non-relativistic contribution). The two scales become comparable when the magnetic field $B$ is $40 \mathrm{~T}$. This results from the Schrödinger terms linear-in- $B$ dependence compared to the $\sqrt{B}$ scaling of the relativistic piece. Therefore, it is important to retain the quadraticin-momentum part of the Hamiltonian when describing the surface states. It has been included in the extensive literature $9,10,20-25$ (both theoretical and experimental) that exists on the phase offset seen in quantum oscillations of the Shubnikov-de Haas and de Haas-van Alphen effects. There are numerous other optical studies in TIs including many in the infrared $26-28$; the most relevant for this work are those in the $\mathrm{THz}$ range $\underline{29,30}$. Here, we mention specifically that of $\mathrm{Wu}$ et al .31 which provides information on the cyclotron frequency itself and, consequently, directly impacts the present work. After the subtraction of a phonon and a lattice polarization term, the cyclotron resonance peak is extracted. It was found that the quadratic-in-momentum term is essential in obtaining a consistent fit to this cyclotron resonance data ${ }^{31}$. In another study of cyclotron resonance data, the Schrödinger piece ${ }^{32}$ was found to be dominant in strained films of $\mathrm{HgTe}$ (an intrinsic TI with negligible bulk carriers ${ }^{33}$ ).

Initially studied TIs where not truly insulating in the bulk and the topologically protected surface states could, thus, be affected by undesirable mixing with the bulk charge carriers. Many strategies have been used to separate out the surface fermions. In the magneto-optical study of $\mathrm{Bi}_{0.91} \mathrm{Sb}_{0.09}$ by Schafgans et al $\stackrel{34}{2}$, several Lan- 
dau level (LL) series are observed. They all display energy dependences that vary as the square root of the applied magnetic field and none are found to originate from the bulk bands since they do not extrapolate to the band gap energy. In $\mathrm{Bi}_{1-x} \mathrm{Sb}_{x}$, the observed quantum oscillations correspond to a two-dimensional Fermi surface and hence, are identified with the surface states 34,35 . They also observe a contribution form a 3D Fermi surface which they assign to coupling between the bulk and surface states.

It has also been possible to separate the surface from the bulk using time-resolved techniques. The surface and bulk states' transient $\mathrm{THz}$ optical response has been found to correspond to very different time scales 36 . Crepaldi et $a l^{37}$ used time-resolved angular-resolved photoemission spectroscopy (ARPES) to differentiate between the two types of states. Such techniques have recently been used ${ }^{38}$ to show that the $A_{1 g}$ phonon mode in $\mathrm{Bi}_{2} \mathrm{Se}_{3}$ is coupled to both the bulk and surface fermions and that this phonon softens on the surface. There are many works on the electron-phonon interaction in TIs (see, for example, Refs ${ }^{39}-41$ ). High resolution ARPES is a particularly useful technique for this kind of investigation. Kinks in the dispersion curves of the charge carriers are interpreted as coupling to possible bosons. Some experiments 41 have given large values for the coupling while others $\$ 9$ give much smaller values. In one study, the coupling was found to be even smaller and interpreted to imply the topological surface states are not only protected from elastic scattering off impurities but also from scattering off low-energy phonons ${ }^{40}$. Such effects are beyond the scope of this paper.

Work has also been done to entirely suppress the metallic character of the bulk states. For example, this can be achieved by doping $\mathrm{Cu}$ in $\mathrm{Bi}_{2} \mathrm{Se}_{3}{ }^{42,43}$. One can then study surface fermions without bulk influences. This is also the case in $\mathrm{Ti}_{1-x} \mathrm{Bi}_{1+x} \mathrm{Se}_{2}$ as documented in the work of Eguchi et al $\underline{\underline{44}}$ and Kuroda et al $\underline{\underline{45}}$.

In the pure relativistic case, there is particle-hole symmetry between the conduction and valence bands which are both described by the same perfect Dirac cone. The introduction of a Schrödinger mass distorts these cones. The conduction band narrows with increasing energy while the valence band fans out as the energy is lowered. This yields an hourglass shape to the overall band structure. The band bending leads to particle-hole asymmetry. In this case, doping with holes rather than electrons will be different than the pure relativistic case. Here, we will emphasize these differences.

When a magnetic field is applied perpendicular to the surface of the TI, Landau levels form. In the pure relativistic system, their energies are characterized by the single Dirac magnetic energy scale $E_{1}=\hbar v_{F} \sqrt{e B / \hbar}$ where $e$ is the elementary charge. When we account for the subdominant non-relativistic contribution, a second Schrödinger magnetic energy scale $E_{0}=\hbar e B / m$ enters and modifies the eigenenergies of the LLs. In particular, the level at zero energy which is so important in the relativistic case is moved to positive energy $\left(E_{0} / 2\right)$. While for small values of $B, E_{1}$ is always larger than $E_{0}$. These two scales become comparable in size as $B$ is increased. This leads to several known effects such as the splitting of the interband absorption lines in the longitudinal conductivity 19.24 . At the same time, the Hall plateaus in the transverse DC conductivity retain $23,24,46$ their relativistic quantization which is distinct from the non-relativistic values which arise when $\hbar e B / m$ is the dominant scale $\underline{46}$.

Even when $B$ is small and the semiclassical regime is considered, for which the chemical potential $|\mu|$ is much greater than both $E_{1}$ and $E_{0}$, important modifications to the cyclotron resonance frequency $\left(\omega_{\mathrm{CR}}\right)$ and its optical spectral weight $\left(W_{\mathrm{CR}}\right)$ arise when $E_{0}$ is introduced. Here we provide a simple analytic formula for the resulting line shape and study the particle-hole asymmetry in both $\omega_{\mathrm{CR}}$ and $W_{\mathrm{CR}}$. While it is the intraband optical transitions which are central to the semiclassical regime, the evolution of the interband transitions in the same limit of $|\mu| \gg E_{1}$ and $E_{0}$ are also of interest. The magnitude of the universal background which emerges is completely independent of the Schrödinger mass but its onset is not 47 . In particular, in this work we emphasize the particle-hole asymmetry of the onset.

The paper is organized as follows: In Sec. II, we introduce the Kubo formula for both the longitudinal and transverse (Hall) magneto-optical conductivity 19,24 . We also present results for both $\operatorname{Re} \sigma_{x x}(\Omega)$ and $\operatorname{Re} \sigma_{x y}(\Omega)$ at several values of $B$ and feature their evolution as $B$ is decreased sufficiently that the LL structures become smooth and reveal a background. In Sec. III, we start from the finite $B$ formulas for the real part of the longitudinal conductivity and show how the semiclassical regime is obtained. The end result is analytic formulae for the cyclotron resonance lineshape, frequency and optical spectral weight. We retain the appropriate limit as $E_{0} \rightarrow 0$. We study the approach to the semiclassical limit and discuss the particle-hole asymmetry that arises. We compare both a constant $|\mu|$ for $\mu \lessgtr 0$ and constant doping level $n$ for electrons and holes. In Sec. IV, we provide a discussion of the universal interband background 47 and its particle-hole asymmetry. An alternative derivation, based on the finite $B$ formula for the conductivity, is provided for the interband background which is additional to the intraband cyclotron resonance line. Details about the asymmetry of the absorption process onsets are provided. Finally, in Sec. V, we give a discussion of our new results and draw conclusions.

\section{FORMALISM}

The model low-energy Hamiltonian on which this work is based includes a small non-relativistic Schrödinger term characterized by a mass $m$ and proportional to the square of the momentum. In addition, we include a dominant relativistic linear-in-momentum Dirac term 
with Fermi velocity $v_{F}$. It has the form ${ }^{48,49}$

$$
\hat{H}=\frac{\hbar^{2} k^{2}}{2 m}+\hbar v_{F}\left(k_{x} \hat{\sigma}_{y}-k_{y} \hat{\sigma}_{x}\right),
$$

where $\hat{\sigma}_{x}$ and $\hat{\sigma}_{y}$ are the Pauli spin matrices and $\boldsymbol{k}$ is the momentum relative to the $\Gamma$ point of the surface state Brillouin zone. As previously discussed, representative values for the mass and velocity are $m \approx 0.1 m_{e}$ (with $m_{e}$ the bare electron mass) and $v_{F}$ of order $10^{5} \mathrm{~m} / \mathrm{s}$, respectively $\underline{18}$. When a magnetic field $B$ is oriented perpendicular to the $2 \mathrm{D}$ surface states, the magnetic energy scales for the relativistic $\left(E_{1}\right)$ and non-relativistic $\left(E_{0}\right)$ pieces of the Hamiltonian are of order $10 \mathrm{meV}$ and $1 \mathrm{meV}$, respectively, for $B=1 \mathrm{~T}$. By definition, $E_{1}=\hbar v_{F} \sqrt{e B / \hbar}$ and $E_{0}=\hbar e B / m$ both involve the magnetic length $l_{B}=\sqrt{\hbar /(e B)}$. In the Landau gauge [with magnetic vector potential $\boldsymbol{A}=(0, B x, 0)]$, the Hamiltonian can be written as

$$
H=\left(\begin{array}{cc}
\frac{\hbar^{2}}{m l_{B}^{2}}\left[a^{\dagger} a+\frac{1}{2}\right] & -\hbar v_{F} \frac{\sqrt{2}}{l_{B}} a \\
-\hbar v_{F} \frac{\sqrt{2}}{l_{B}} a^{\dagger} & \frac{\hbar^{2}}{m l_{B}^{2}}\left[a^{\dagger} a+\frac{1}{2}\right]
\end{array}\right),
$$

where

$$
a^{\dagger} \equiv \frac{l_{B}}{\sqrt{2}}\left[-i k_{x}+\frac{x+k_{y} l_{B}^{2}}{l_{B}^{2}}\right]
$$

and

$$
a \equiv \frac{l_{B}}{\sqrt{2}}\left[i k_{x}+\frac{x+k_{y} l_{B}^{2}}{l_{B}^{2}}\right]
$$

are the creation and annihilation operators of the harmonic oscillator Hamiltonian. The energy of the LLs is

$$
\mathcal{E}_{N, s}=\left\{\begin{array}{cc}
E_{0} N+s \sqrt{2 N E_{1}^{2}+\frac{E_{0}^{2}}{4}} & N=1,2,3, \ldots \\
\frac{E_{0}}{2} & N=0
\end{array},\right.
$$

where $s= \pm$ for the conduction and valence band, respectively. A schematic comparison between the LLs of the pure relativistic limit $\left(E_{0}=0\right)$ and TI is shown in Fig. 1. The LLs (dots) are overlaid on the respective $B=0$ band structures. In the TI, the $N=0$ level is no longer pinned at zero energy but has shifted upwards. The symmetry between the conduction (red) and valence (blue) levels is also lost with finite $E_{0}$. In the following numerical work, we will take $E_{1} / \sqrt{B}=10.4 \mathrm{meV} / \sqrt{\mathrm{T}}$ and $E_{0} / B=1.1 \mathrm{meV} / \mathrm{T}$ as characteristic values of a TI. In terms of the spin-up and spin-down amplitudes, the eigenfunctions of the state $|N s\rangle$ are

$$
|N s\rangle=\left(\begin{array}{c}
\mathcal{C}_{N, s}^{\uparrow}|N-1\rangle \\
\mathcal{C}_{N, s}^{\downarrow}|N\rangle
\end{array}\right)
$$

Pure Relativistic

Topological Insulator

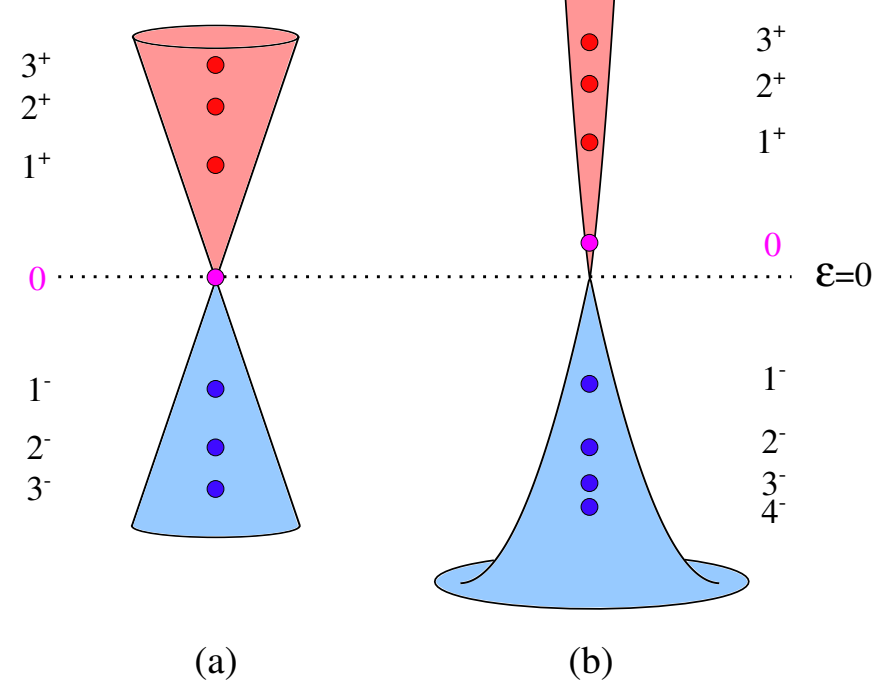

FIG. 1. (Color online) (a) Perfect Dirac surface states in the pure relativistic limit. (b) Hourglass shaped spectrum when a subdominant non-relativistic term is present. The solid dots with labels $0,1^{ \pm}, 2^{ \pm}, \ldots$ show the energy of the LLs in a magnetic field. The shading indicates the conduction (red) and valence (blue) bands.

where $a|N\rangle=\sqrt{N}|N-1\rangle$ and $a^{\dagger}|N\rangle=$ $\sqrt{N+1}|N+1\rangle$. The spin-up amplitude is

$$
\mathcal{C}_{N, s}^{\uparrow}=\left\{\begin{array}{cc}
-s \sqrt{\frac{1}{2}-s \frac{E_{0} / 2}{2\left(\mathcal{E}_{N,+}-E_{0} N\right)}} & N=1,2,3, \ldots \\
0 & N=0
\end{array},\right.
$$

and

$$
\mathcal{C}_{N, s}^{\downarrow}=\left\{\begin{array}{cc}
\sqrt{\frac{1}{2}+s \frac{E_{0} / 2}{2\left(\mathcal{E}_{N,+}-E_{0} N\right)}} & N=1,2,3, \ldots \\
1 & N=0
\end{array},\right.
$$

gives the spin-down component.

Using the Kubo formula, the dynamical conductivity $\sigma_{\alpha \beta}(\Omega)$ is given by

$$
\begin{aligned}
\sigma_{\alpha \beta}(\Omega)=\frac{i \hbar}{2 \pi l_{B}^{2}} \sum_{\substack{N, M=0 \\
s, s^{\prime}= \pm}}^{\infty} \frac{f_{M, s^{\prime}}-f_{N, s}}{\mathcal{E}_{N, s}-\mathcal{E}_{M, s^{\prime}}} \\
\quad \times \frac{\left\langle\bar{N} s\left|\hat{j}_{\alpha}\right| \bar{M} s^{\prime}\right\rangle\left\langle\bar{M} s^{\prime}\left|\hat{j}_{\beta}\right| \bar{N} s\right\rangle}{\hbar \Omega+\mathcal{E}_{M, s^{\prime}}-\mathcal{E}_{N, s}+i \hbar /(2 \tau)},
\end{aligned}
$$

where $f_{N, s}$ is the Fermi function for state $N$ in band $s, \alpha$ and $\beta$ index the $x$ and $y$ components, $\tau$ is a phenomenological optical scattering time taken to be a large constant and $\hat{j}_{\alpha}=e \hat{v}_{\alpha} \equiv(e / \hbar)\left(\partial \hat{H} / \partial k_{\alpha}\right)$ is the current operator. At zero temperature, $f_{N, s}$ can be replaced by 
the Heaviside step function $\Theta\left(\mu-\mathcal{E}_{N, s}\right)$; here, $\mu$ is the chemical potential. The necessary velocity operators are

$$
\hat{v}_{x}=\frac{\hbar}{m} k_{x}+v_{F} \hat{\sigma}_{y}=i \frac{\hbar}{m} \frac{a^{\dagger}-a}{\sqrt{2} l_{B}}+v_{F} \hat{\sigma}_{y},
$$

and

$$
\hat{v}_{y}=\frac{\hbar}{m}\left[k_{y}+\frac{e B x}{\hbar}\right]-v_{F} \hat{\sigma}_{x}=\frac{\hbar}{m} \frac{a^{\dagger}+a}{\sqrt{2} l_{B}}-v_{F} \hat{\sigma}_{x} .
$$

Calculating the appropriate matrix elements, we obtain the real part of the longitudinal conductivity 24

$$
\begin{aligned}
\operatorname{Re}\left\{\frac{\sigma_{x x}(\Omega)}{e^{2} / \hbar}\right\} & =\frac{E_{1}^{2}}{2 \pi} \sum_{\substack{N, M=0 \\
s, s^{\prime}= \pm}}^{\infty} \frac{f_{M, s^{\prime}}-f_{N, s}}{\mathcal{E}_{N, s}-\mathcal{E}_{M, s^{\prime}}} \\
& \times \frac{\eta}{\left(\Omega+\mathcal{E}_{M, s^{\prime}}-\mathcal{E}_{N, s}\right)^{2}+\eta^{2}} \\
& \times\left[\mathcal{F}\left(N s ; M s^{\prime}\right) \delta_{N, M-1}+\mathcal{F}\left(M s^{\prime} ; N s\right) \delta_{M, N-1}\right]
\end{aligned}
$$

where $\eta \equiv \hbar /(2 \tau)$ is a constant phenomenological optical scattering rate and ${ }^{24}$

$$
\begin{aligned}
\mathcal{F}\left(N s ; M s^{\prime}\right) & \equiv\left[\mathcal{C}_{M, s^{\prime}}^{\uparrow} \mathcal{C}_{N, s}^{\downarrow}-\frac{E_{0}}{\sqrt{2} E_{1}}\right. \\
& \left.\times\left(\sqrt{N} \mathcal{C}_{M, s^{\prime}}^{\uparrow} \mathcal{C}_{N, s}^{\uparrow}+\sqrt{N+1} \mathcal{C}_{M, s^{\prime}}^{\downarrow} \mathcal{C}_{N, s}^{\downarrow}\right)\right]^{2}
\end{aligned}
$$

The imaginary part of the conductivity is found by replacing the $\eta$ factor in the numerator with $\Omega+\mathcal{E}_{M, s^{\prime}}-$ $\mathcal{E}_{N, s}$. Similarly, the real part of the transverse Hall conductivity is 24

$$
\begin{aligned}
\operatorname{Re}\left\{\frac{\sigma_{x y}(\Omega)}{e^{2} / \hbar}\right\} & =\frac{E_{1}^{2}}{2 \pi} \sum_{\substack{N, M=0 \\
s, s^{\prime}= \pm}}^{\infty} \frac{f_{M, s^{\prime}}-f_{N, s}}{\mathcal{E}_{N, s}-\mathcal{E}_{M, s^{\prime}}} \\
& \times \frac{\Omega+\mathcal{E}_{M, s^{\prime}}-\mathcal{E}_{N, s}}{\left(\Omega+\mathcal{E}_{M, s^{\prime}}-\mathcal{E}_{N, s}\right)^{2}+\eta^{2}}
\end{aligned}
$$$$
\times\left[\mathcal{F}\left(N s ; M s^{\prime}\right) \delta_{N, M-1}-\mathcal{F}\left(M s^{\prime} ; N s\right) \delta_{M, N-1}\right]
$$

Here, the imaginary part is given by the replacement $\Omega+$ $\mathcal{E}_{M, s^{\prime}}-\mathcal{E}_{N, s} \rightarrow-\eta$ in the numerator.

\section{NUMERICAL RESULTS FOR THE DYNAMICAL CONDUCTIVITY}

To set the stage for understanding the semiclassical limit, we start by examining how the dynamical conductivity evolves as the magnitude of the magnetic field $B$ is progressively decreased towards $B=0$. As we are interested in modifications to the semiclassical limit brought about by the subdominant non-relativistic (Schrödinger) contribution in the Hamiltonian [Eqn. (1)], differences between the pure relativistic (Dirac) and TI are emphasized.

In Fig. 2, we compare $\operatorname{Re} \sigma_{x x}(\Omega)$ for a typical TI with the ideal relativistic system. The top frame is for $E_{0}=0$

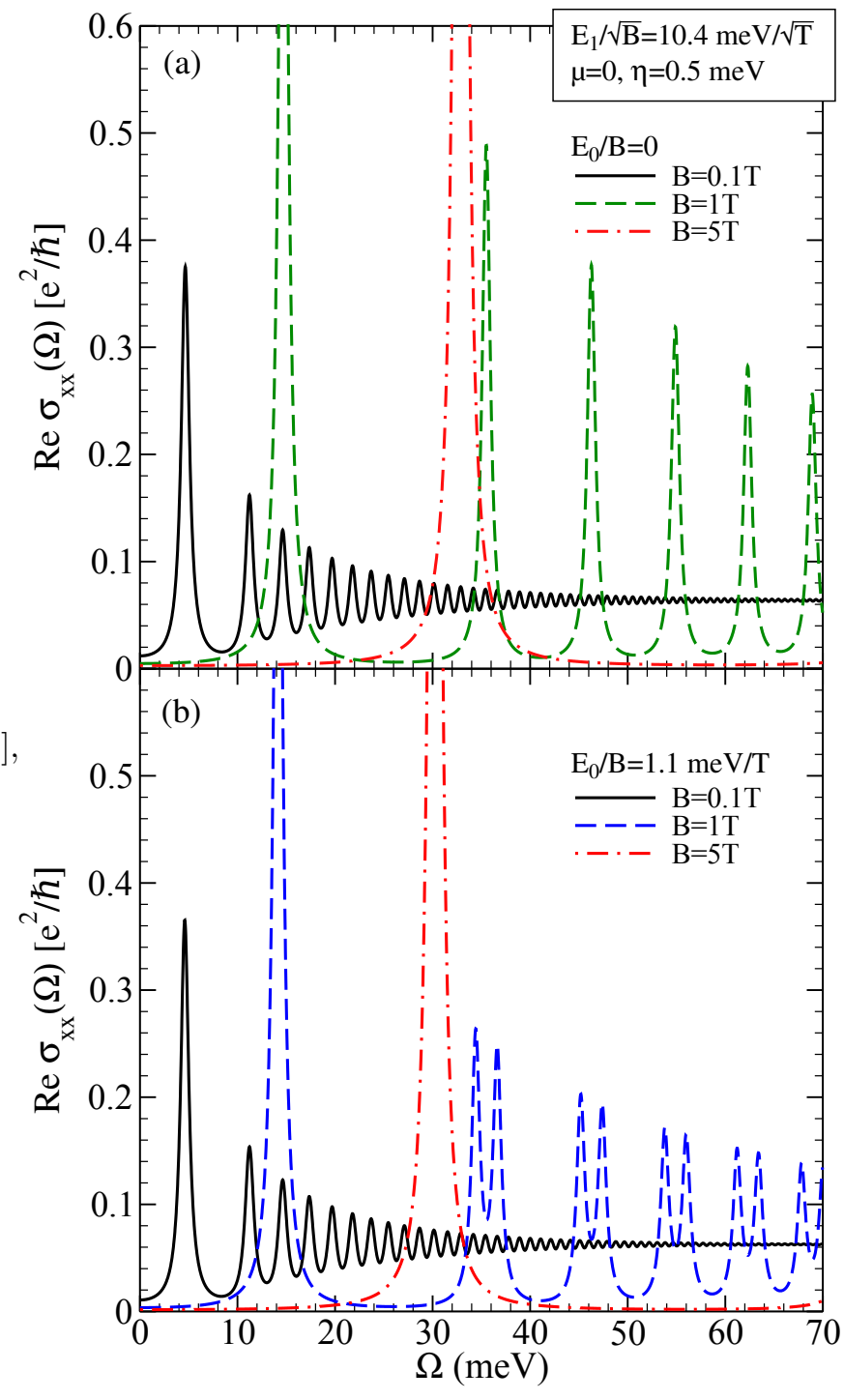

FIG. 2. (Color online) Real part of the longitudinal optical . conductivity as a function of photon energy at charge neutrality $(\mu=0)$ for (a) the pure relativistic case and (b) with the addition of a non-relativistic contribution. Three values of $B$ are considered: $B=0.1 \mathrm{~T}, B=1 \mathrm{~T}$ and $B=5 \mathrm{~T}$.

(i.e. no Schrödinger mass term). In both cases, we take the Dirac magnetic energy scale to be $E_{1} / \sqrt{B}=10.4$ $\mathrm{meV} / \sqrt{\mathrm{T}}$ and set the chemical potential at charge neutrality $(\mu=0)$. Three values of magnetic field are considered: $\mathrm{B}=0.1 \mathrm{~T}$ (solid black curve), $\mathrm{B}=1 \mathrm{~T}$ [dashed green/blue curve for (a)/(b)] and $\mathrm{B}=5 \mathrm{~T}$ (dash-dotted red curve). Therefore, $E_{1}=3.29 \mathrm{meV}, 10.5 \mathrm{meV}$ and 23.3 meV, respectively. It is these energies that determine the energies of the various LLs. For $E_{0}=0$, Eqn. (5) gives $\mathcal{E}_{N, s}=s \sqrt{2 N E_{1}}$ for $N=1,2,3, \ldots$ and $\mathcal{E}_{0}=0$ for $N=0$. In the pure relativistic case, one has perfect particle-hole symmetry about the neutrality point. In the numerical work, we have taken the optical scattering rate $\eta$ of Eqn. (12) to be $0.5 \mathrm{meV}$ which provides 
a width to the optical absorption lines that otherwise correspond to Dirac $\delta$-functions. First, we note that a universal interband background becomes well defined at high photon frequencies in the solid black curve. In units of $e^{2} / \hbar$, this takes the value of $1 / 16$. For larger magnetic fields (dashed blue and dash-dotted red curves), the energy spacing between the optical peaks has increased and the background is no longer resolved. In all cases, the first line is an intraband transition from $N=0$ to $(N, s)=(1,+)$ while all other lines are due to interband transitions from $(N,-) \rightarrow(N \pm 1,+)$ with $N=1,2,3, \ldots$. The two interband lines for a given $N$ are degenerate in energy for a given value of $N$. These results are for comparison with those presented in the lower frame which apply to the case when the Schrödinger energy $E_{0}$ is not set at zero $\left(E_{0} / B=1.1 \mathrm{meV} / \mathrm{T}\right)$. For $B=0.1 \mathrm{~T}, E_{0}$ is small compared to $E_{1}$. For $B=5 \mathrm{~T}$, it has increased to $5.5 \mathrm{meV}$ which is roughly a factor of four smaller than $E_{1}$. Still, the energies at which the various lines occur have not shifted a great deal when compared to the top frame; however, we can clearly see that the intraband line for $B=5 \mathrm{~T}$ has shifted downward upon the introduction of a Schrödinger term. As is clearly seen in the dashed blue curve, for $B=1 \mathrm{~T}$, the interband transition peak has split into two with the energy of the splitting given by the value of $E_{0}$. This can be traced to the fact that, in a TI, the lines $(N,-) \rightarrow(N+1,+)$ and $(N,-) \rightarrow(N-1,+)$ are no longer degenerate in energy. As $B$ is reduced in magnitude, the splitting of the interband peaks into doublets is no longer resolved (see the solid black curve for $B=0.1 \mathrm{~T})$. This is because $E_{0}$ itself becomes small and the residual scattering rate that we have used $(\eta=0.5$ meV) washes out the splitting. The universal background seen at higher values of photon energy $\Omega$ rapidly becomes constant and equal to $e^{2} /(16 \hbar)$, completely independent of the Schrödinger mass term. In fact, because we have taken $\mu=0$ (charge neutrality), the entire curve becomes constant independent of $\Omega$ for $B=0$.

It is illuminating to also consider the evolution of the Hall conductivity as $B \rightarrow 0$. We know that at $B=0$, this quantity $\left[\operatorname{Re} \sigma_{x y}(\Omega)\right]$ must be zero rather than the constant value seen above for $\operatorname{Re} \sigma_{x x}(\Omega)$. In Fig. 3, we provide a comparison between the pure Dirac and TI for the real part of the Hall conductivity. The same three values of magnetic field are considered and the notation is the same as for Fig. 2. Again, the energy of the first peak/valley is only slightly affected by the introduction of an $E_{0}$ term. However, there are important differences which must be noted. Namely, in the top frame, the peak is always first followed by a valley at slightly higher energy. In the bottom frame, it is the opposite. Associated with this reversal is the fact that the DC limit of $\operatorname{Re} \sigma_{x y}(\Omega)$ which gives the quantized Hall conductivity has the same magnitude $(1 /(4 \pi)$ in our units) but is of opposite sign. This change has been brought about by the introduction of the Schrödinger term $E_{0}$; this does not change the quantization in the plateau height (which remains relativistic) but does change the energy (chemi-

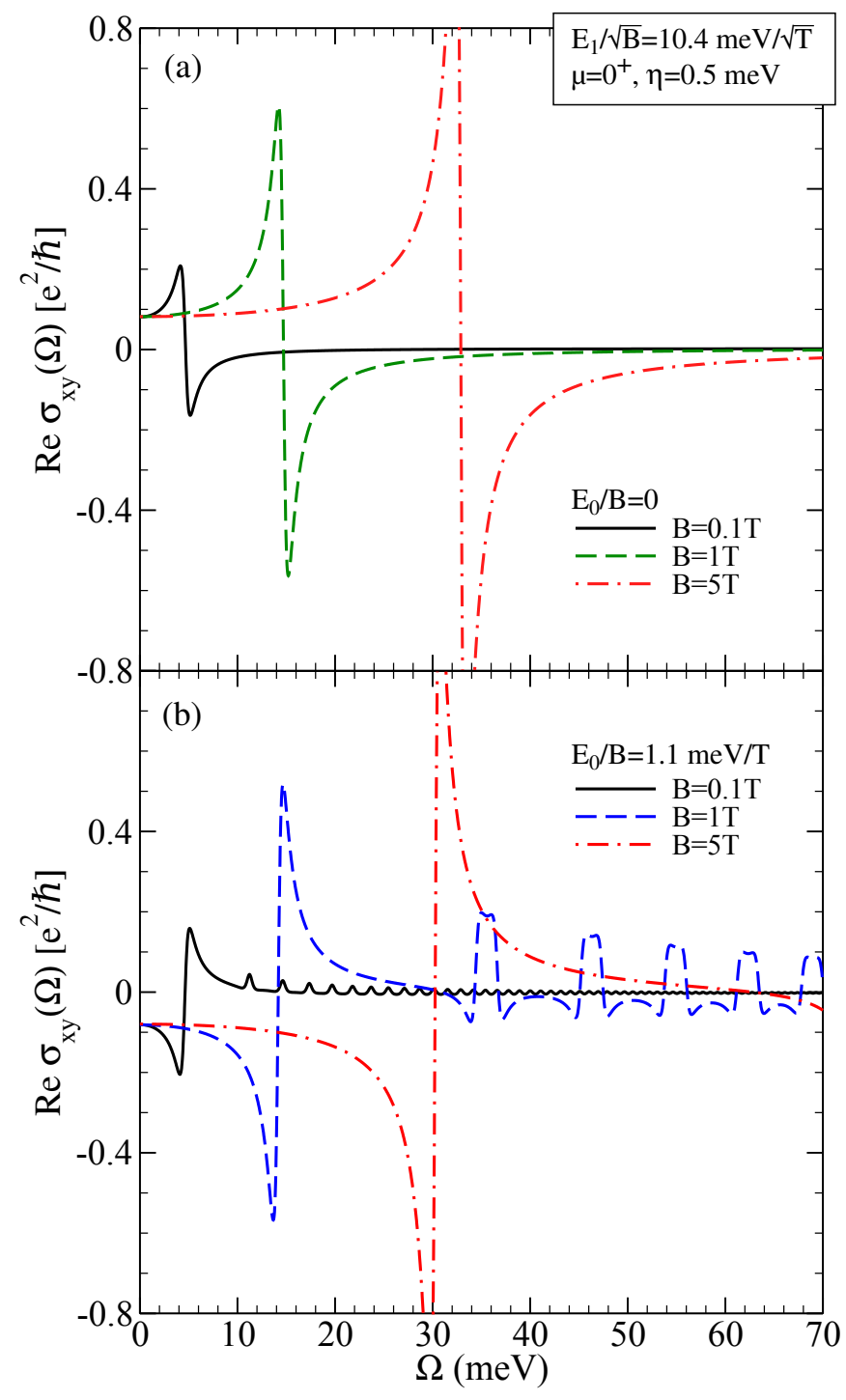

FIG. 3. (Color online) Real part of the transverse Hall conductivity for the same parameters as Fig. 2 .

cal potential) at which the step to a new plateau occurs. For a TI, the step from $-1 / 2$ to $1 / 2$ quantization (in units of $e^{2} / h$ ) occurs at finite $\mu$ rather than at $\mu=0^{+}$. This is due to the first LL sitting at an energy $E_{0} / 2$ rather than at zero. Some further explanation of our language is required. As we have stated, in our units, the universal interband background has a value of $1 / 16$ or $\operatorname{Re} \sigma_{x x}(\Omega)=e^{2} /(16 \hbar)=e^{2} \pi /(8 h)$ which is a factor of four smaller than the well known value of $e^{2} \pi /(2 h)$ for graphene. In graphene, this value accounts for a valleyspin degeneracy which is not present in a TI. Further, the Hall plateau quantization is $\pm 1 / 2, \pm 3 / 2, \pm 5 / 2, \ldots$ in graphene (in units of $e^{2} / h$ ) if the four fold degeneracy is removed. Another striking difference between the pure relativistic [frame (a)] and the TI case [frame (b)] is the additional LL structure seen in the lower frame which is absent in the upper frame. This is traced to the split- 
ting of the optical transitions between $N-1 \rightarrow N$ and $N \rightarrow N+1$ for a TI. These oscillations are clearly significant at larger values of $B$ but are progressively reduced as $B$ becomes small. Note that for the $B=0.1 \mathrm{~T}$ (solid black curve), $\operatorname{Re} \sigma_{x y}(\Omega) \sim 0$ for most of the $\Omega$ range shown. At $B=0$, the entire curve is of course zero.

So far, we have considered $\mu=0$ to get a first understanding of the differences and unchanged characteristics of the magneto-optics which are brought about by the introduction of a subdominant Schrödinger piece in the Hamiltonian. Next, we will concentrate on finite $\mu$. In fact, our interest will be on the semiclassical regime for $\mu$ much larger than the magnetic scales $E_{1}$ and $E_{0}$. In Fig. 4, we show the results for the real parts of the longitudinal conductivity $\operatorname{Re} \sigma_{x x}(\Omega)$ vs. $\Omega$ [frame (a)] and the Hall conductivity $\operatorname{Re} \sigma_{x y}(\Omega)$ vs. $\Omega$ [frame (b)]. The magnetic field is set at $B=0.2 \mathrm{~T}$ and three values of $\mu$ are considered. The solid black curve corresponds to $\mu=0$ and is for comparison. The dashed red curve applies to $\mu=8 \mathrm{meV}$ and the dash-dotted green line is for $\mu=10$ meV. We want to focus on the first intraband peak and understand its evolution as $\mu$ is increased. It is this line which eventually evolves into the cyclotron resonance line of the semiclassical theory. We note that, as $\mu$ increases, the position of this peak moves to lower energies and acquires added intensity (optical spectral weight). Frame (b) gives $\operatorname{Re} \sigma_{x y}(\Omega)$. We note that, in these curves, the position in energy of the first valley-to-peak (for $\mu=0$ ) and peak-to-valley (for finite $\mu$ ) structures also evolves towards lower energies and tracks the intraband peaks in $\operatorname{Re} \sigma_{x x}(\Omega)$ of the top frame. In particular, we emphasize the DC value of $\operatorname{Re} \sigma_{x y}(\Omega)$ which gives the Hall plateaus. For $\mu=0$, it is $-1 / 2$ [i.e. $-e^{2} /(4 \pi \hbar)$ ] while for $\mu=8$ $\mathrm{meV}$ it has moved to $3 / 2$ and then to $5 / 2$ (in units of $\left.e^{2} / h\right)$ for $\mu=10 \mathrm{meV}$. These values are independent of the mass term in the Hamiltonian. We now turn to the semiclassical regime $\mu \gg E_{1}$ and $E_{0}$.

\section{SEMICLASSICAL LIMIT}

We begin with Eqn. (12) for the real part of the AC longitudinal conductivity. We work in the limit of $\mu$ much larger than the magnetic quantization such that the chemical potential falls between the $N_{c}^{\text {th }}$ and $\left(N_{c}+1\right)^{\text {th }}$ LLs with $N_{c}$ very large and given by

$$
\mu=E_{0} N_{c}+\sqrt{2 N_{c} E_{1}^{2}+\left(\frac{E_{0}}{2}\right)^{2}}
$$

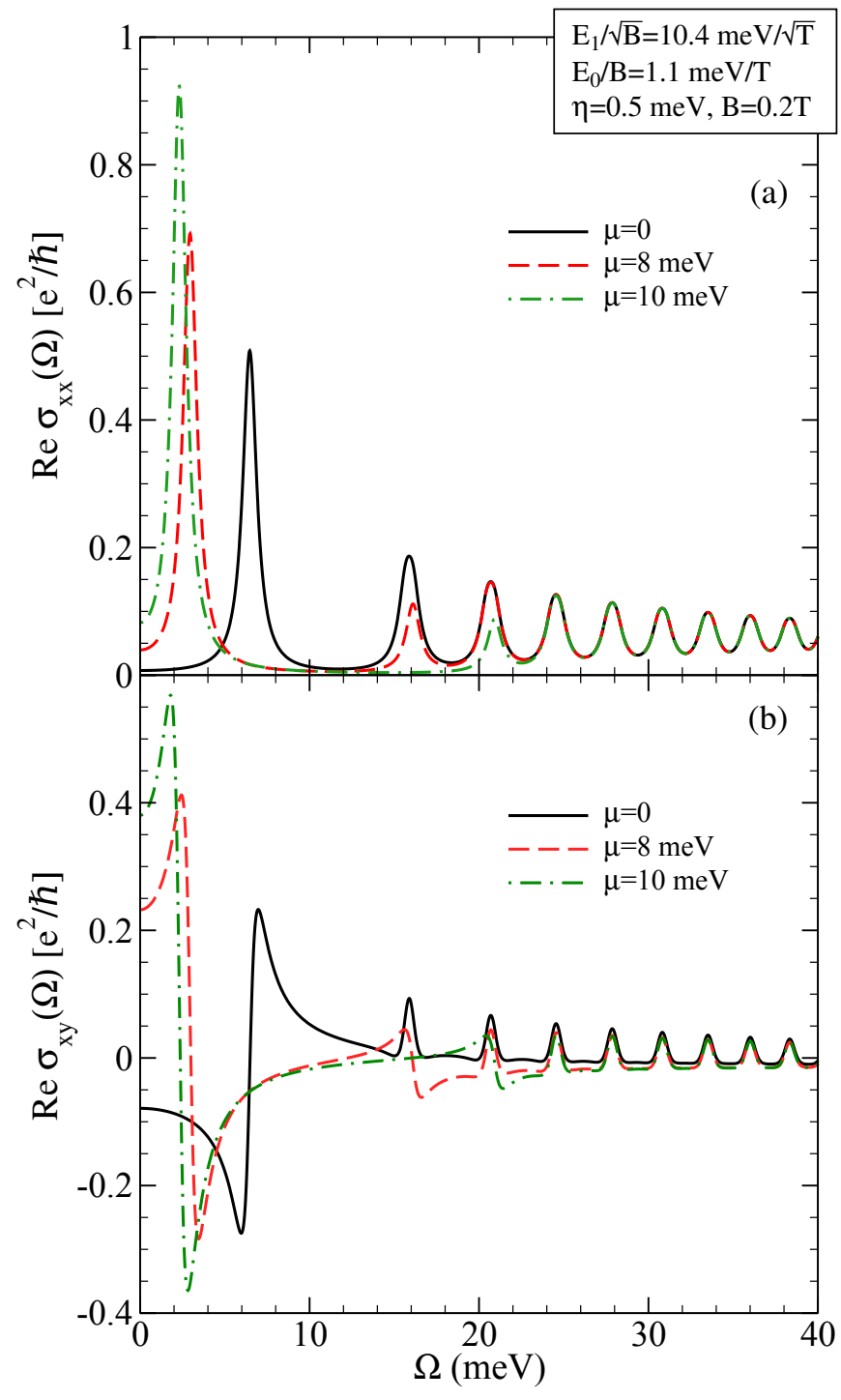

FIG. 4. (Color online) (a) Real part of the longitudinal AC conductivity as a function of photon energy for $B=0.2 \mathrm{~T}$ and three values of chemical potential: $\mu=0$ (charge neutrality), $\mu=8 \mathrm{meV}$ and $\mu=10 \mathrm{meV}$. (b) Real part of the Hall conductivity for the same parameters as (a).

For definiteness, we assume $\mu>0$ (i.e. the conduction band is partially filled). In this case

$$
\begin{aligned}
\operatorname{Re}\left\{\frac{\sigma_{x x}(\Omega)}{e^{2} / \hbar}\right\} & =\frac{E_{1}^{2}}{2 \pi} \sum_{N=0}^{\infty} \frac{f_{N,+}-f_{N-1,+}}{\mathcal{E}_{N-1,+}-\mathcal{E}_{N,+}} \\
& \times\left\{\frac{\eta \mathcal{F}(N-1+; N+)}{\left(\Omega+\mathcal{E}_{N,+}-\mathcal{E}_{N-1,+}\right)^{2}+\eta^{2}}\right. \\
& \left.+\frac{\eta \mathcal{F}(N-1+; N+)}{\left(\Omega+\mathcal{E}_{N-1,+}-\mathcal{E}_{N,+}\right)^{2}+\eta^{2}}\right\} .
\end{aligned}
$$

From here on, the + index shall be implied (and suppressed) as we are only interested in $s, s^{\prime}=+$. In our limit, the first factor of the sum becomes $-\partial f_{N} / \partial \mathcal{E}_{N}$ and 
the energy difference $\mathcal{E}_{N}-\mathcal{E}_{N-1} \equiv \omega_{\mathrm{CR}}(\mu)$ is the cyclotron resonance energy for $\mu>0$. At zero temperature,

$$
\begin{aligned}
& \operatorname{Re}\left\{\frac{\sigma_{x x}(\Omega)}{e^{2} / \hbar}\right\}=\frac{E_{1}^{2}}{2 \pi} \sum_{N=0}^{\infty} \delta\left(\mu-\mathcal{E}_{N}\right) \mathcal{F}(N-1 ; N) \\
& \quad \times\left\{\frac{\eta}{\left(\Omega+\omega_{\mathrm{CR}}\right)^{2}+\eta^{2}}+\frac{\eta}{\left(\Omega-\omega_{\mathrm{CR}}\right)^{2}+\eta^{2}}\right\} .
\end{aligned}
$$

For $B \rightarrow 0, E_{0} \ll E_{1}$ and Eqn. (15) gives

$$
\sqrt{2 N_{c}}=\frac{E_{1}}{E_{0}}\left\{\sqrt{1+\frac{2 \mu}{m v_{F}^{2}}}-1\right\} .
$$

The sum over $N$ in Eqn. (17) also changes into an integral. Therefore, the Dirac $\delta$-function $\delta\left(\mu-\mathcal{E}_{N}\right)$ means that the entire integrand is to be evaluated at $N=N_{c}$. This gives

$$
\begin{aligned}
\operatorname{Re} & \left\{\frac{\sigma_{x x}(\Omega)}{e^{2} / \hbar}\right\}=\frac{E_{1}^{2}}{2 \pi} \frac{1}{\partial \mathcal{E}_{N} /\left.\partial N\right|_{N_{c}}} \mathcal{F}\left(N_{c}-1 ; N_{c}\right) \\
& \times\left\{\frac{\eta}{\left(\Omega+\omega_{\mathrm{CR}}\right)^{2}+\eta^{2}}+\frac{\eta}{\left(\Omega-\omega_{\mathrm{CR}}\right)^{2}+\eta^{2}}\right\} .
\end{aligned}
$$

But

$$
\left.\frac{\partial \mathcal{E}_{N}}{\partial N}\right|_{N_{c}}=\frac{E_{0} \sqrt{1+\frac{2 \mu}{m v_{F}^{2}}}}{\sqrt{1+\frac{2 \mu}{m v_{F}^{2}}}-1}
$$

and hence

$$
\begin{aligned}
\operatorname{Re} & \left\{\frac{\sigma_{x x}(\Omega)}{e^{2} / \hbar}\right\}=\frac{m v_{F}^{2}}{2} \frac{\sqrt{1+\frac{2 \mu}{m v_{F}^{2}}}-1}{\sqrt{1+\frac{2 \mu}{m v_{F}^{2}}}} \mathcal{F}\left(N_{c}-1 ; N_{c}\right) \\
& \times\left\{\frac{\eta / \pi}{\left(\Omega+\omega_{\mathrm{CR}}\right)^{2}+\eta^{2}}+\frac{\eta / \pi}{\left(\Omega-\omega_{\mathrm{CR}}\right)^{2}+\eta^{2}}\right\} .
\end{aligned}
$$

The only $\Omega$ dependence that remains is in the curly bracket and the integral over photon energy of this term is normalized to a value of one. It provides an analytic formula for the frequency profile of the cyclotron resonance line. The remaining factors in Eqn. (21) give the optical spectral weight of the cyclotron resonance which we denote $W_{C R}$, where

$$
W_{C R}=\frac{e^{2}}{\hbar} \frac{m v_{F}^{2}}{2} \frac{\sqrt{1+\frac{2 \mu}{m v_{F}^{2}}}-1}{\sqrt{1+\frac{2 \mu}{m v_{F}^{2}}}} \mathcal{F}\left(N_{c}-1 ; N_{c}\right) \text {. }
$$

We now need to evaluate $\mathcal{F}\left(N_{c}-1 ; N_{c}\right)$. Since $N_{c}$ is very large as $B \rightarrow 0$, we can replace $N_{c}-1$ by $N_{c}$ to get

$$
\begin{aligned}
\mathcal{F}\left(N_{c} ; N_{c}\right) & \approx\left[\mathcal{C}_{N_{c}}^{\uparrow} \mathcal{C}_{N_{c}}^{\downarrow}-\frac{E_{0}}{\sqrt{2} E_{1}}\right. \\
& \left.\times\left(\sqrt{N_{c}} \mathcal{C}_{N_{c}}^{\uparrow} \mathcal{C}_{N_{c}}^{\uparrow}+\sqrt{N_{c}} \mathcal{C}_{N_{c}}^{\downarrow} \mathcal{C}_{N_{c}}^{\downarrow}\right)\right]^{2}
\end{aligned}
$$

which follows from Eqn. (13). Using Eqns. (7) and (8), we note that

$$
\mathcal{C}_{N_{c}}^{\uparrow} \approx-\sqrt{\frac{1}{2}-\frac{E_{0} / 2}{2 \sqrt{2 N_{c}} E_{1}}}
$$

and

$$
\mathcal{C}_{N_{c}}^{\downarrow} \approx \sqrt{\frac{1}{2}+\frac{E_{0} / 2}{2 \sqrt{2 N_{c}} E_{1}}}
$$

Using Eqn. (18) for $\sqrt{2 N_{c}}$, we find

$$
\frac{E_{0}}{\sqrt{2 N_{c}} E_{1}}=\frac{E_{0}}{m v_{F}^{2}} \frac{1}{\sqrt{1+\frac{2 \mu}{m v_{F}^{2}}}-1}
$$

which goes to zero as $B \rightarrow 0$. Therefore, $\mathcal{C}_{N_{c}}^{\uparrow} \approx-\sqrt{1 / 2}$ and $\mathcal{C}_{N_{c}}^{\downarrow} \approx \sqrt{1 / 2}$. Thus

$$
\mathcal{F}\left(N_{c} ; N_{c}\right) \approx\left(-\frac{1}{2}-\frac{E_{0}}{\sqrt{2} E_{1}} \sqrt{N_{c}}\right)^{2}=\frac{1}{4}\left(1+\frac{2 \mu}{m v_{F}^{2}}\right)
$$

When this is substituted into Eqn. (22), we obtain

$$
W_{C R}=\frac{e^{2}}{\hbar} \frac{m v_{F}^{2}}{8}\left[\sqrt{1+\frac{2 \mu}{m v_{F}^{2}}}-1\right] \sqrt{1+\frac{2 \mu}{m v_{F}^{2}}}
$$

for the optical spectral weight of the cyclotron resonance line. Including negative chemical potentials, we find

$$
W_{C R}=\frac{e^{2}}{\hbar} \frac{m v_{F}^{2}}{8}\left|\sqrt{1+\frac{2 \mu}{m v_{F}^{2}}}-1\right| \sqrt{1+\frac{2 \mu}{m v_{F}^{2}}}
$$

for $\mu \lessgtr 0$. The profile in photon energy of the line is given by

$$
\frac{\eta / \pi}{\left(\Omega+\omega_{\mathrm{CR}}\right)^{2}+\eta^{2}}+\frac{\eta / \pi}{\left(\Omega-\omega_{\mathrm{CR}}\right)^{2}+\eta^{2}}
$$

for both $\mu \lessgtr 0$. This formula is unchanged from the result found for graphene ${ }^{50}$ except that the cyclotron frequency $\omega_{\mathrm{CR}}$ has been altered. By definition, the cyclotron resonance energy $\omega_{\mathrm{CR}}$ at chemical potential $\mu$ is given by $\mathcal{E}_{N_{c}}-\mathcal{E}_{N_{c}-1} \equiv \omega_{\mathrm{CR}}$ in the limit of large $N_{c}$ determined by $\mu$. We get

$$
\omega_{\mathrm{CR}}(\mu)=\frac{\hbar e B v_{F}^{2}}{2|\mu|}\left(1+\sqrt{1+\frac{2 \mu}{m v_{F}^{2}}}\right) \sqrt{1+\frac{2 \mu}{m v_{F}^{2}}}
$$

which does depend on the Schrödinger mass. In Fig. 15(a), we plot the optical spectral weight $\left(W_{C R}\right)$ of the cyclotron resonance line in a TI as a function of $|\mu|$. Three cases are considered. The dash-dotted green curve is for comparison and corresponds to the pure relativistic limit. In this instance, $W_{C R}$ increases linearly with $|\mu|$. For a TI with finite Schrödinger contribution, the red curve 

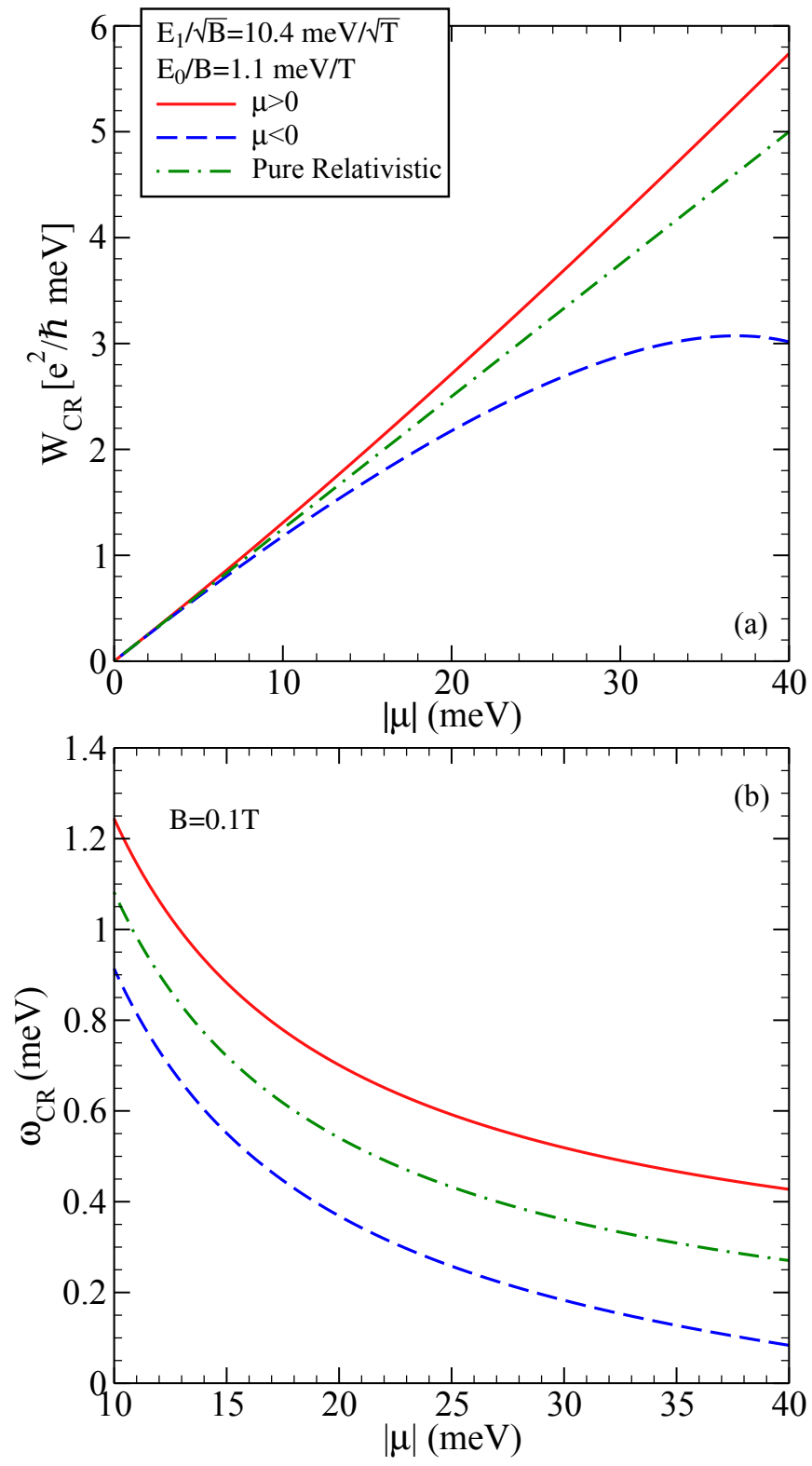

FIG. 5. (Color online) (a) Optical spectral weight under the cyclotron resonance line as a function of $|\mu|$ for the pure relativistic and TI regimes. (b) The corresponding results for the cyclotron frequency at $B=0.1 \mathrm{~T}$.

$(\mu>0)$ is always above the relativistic case while the dashed blue curve $(\mu<0)$ is always below it. In frame (b), we present the complimentary results for the $|\mu|$ dependence of the cyclotron resonance frequency $\omega_{\mathrm{CR}}$ for $B=0.1 \mathrm{~T}$. Again, we see deviations from the pure relativistic limit with the solid red curve $(\mu>0)$ and dashdotted blue curve $(\mu<0)$ falling above and below this case (dash-dotted green), respectively. It is interesting to obtain a first correction to $W_{C R}$ and $\omega_{\mathrm{CR}}$ as a function of $1 / m$. Trivially, the $m \rightarrow \infty$ limit of Eqn. (31) gives the well known result for $\omega_{\mathrm{CR}}$ in the pure relativistic regime; that is, $\omega_{\mathrm{CR}}=\hbar e B v_{F}^{2} /|\mu|$. The first correction is a factor of

$$
\omega_{\mathrm{CR}}^{1}(\mu)=1+\frac{3 \mu}{2 m v_{F}^{2}}
$$

which, as seen in the Fig. 5(b), moves the curve up for $\mu>0$ and down for $\mu<0$. In Fig. 5(b), only $|\mu|>10$ $\mathrm{meV}$ is shown as cyclotron resonance is defined for large $\mu$. For the optical spectral weight, the first correction gives

$$
W_{C R} \approx \frac{e^{2}}{8 \hbar} \mu\left(1+\frac{\mu}{2 m v_{F}^{2}}\right) .
$$

Again, the additional contribution to the relativistic limit is positive (negative) for $\mu>0(\mu<0)$.

So far, the results have been presented as a function of chemical potential. Of course, the electronic density of states in a TI is not symmetric with respect to the neutrality (Dirac) point. It rises above the pure Dirac limit for negative energies and is reduced below this regime for positive $\mu$. This is shown in the inset of Fig. 6. Thus, for

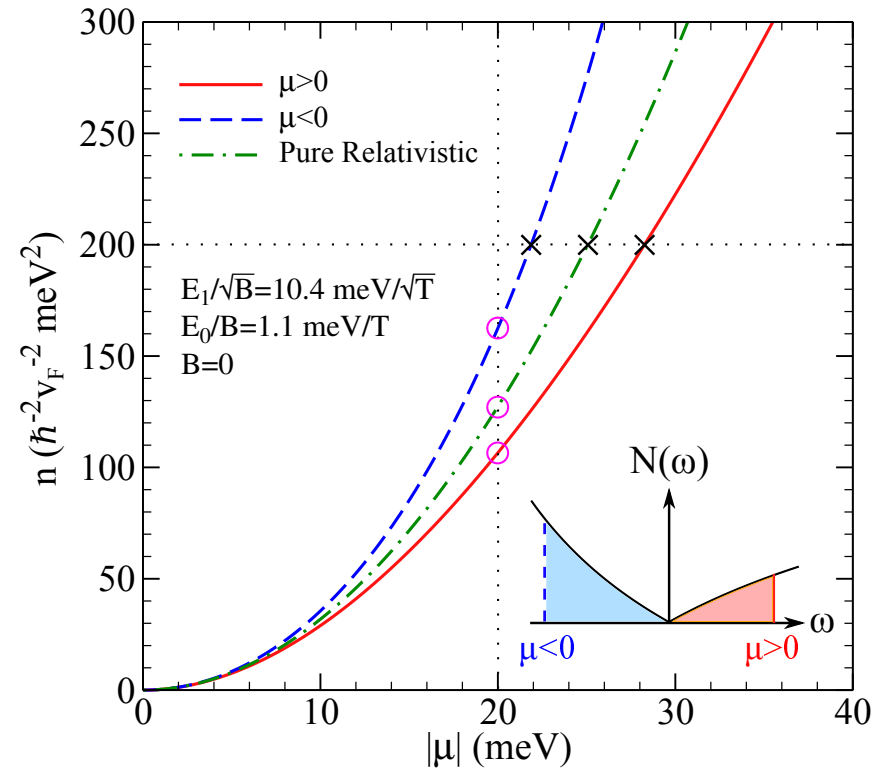

FIG. 6. (Color online) The number of dopants $(n)$ as a function of the magnitude of the chemical potential. The pure relativistic case is contrasted with that when a subdominant Schrödinger term is included. Inset: density of states as a function of $\omega$. The shading identifies the conduction (red) and valence (blue) bands.

a given magnitude of $\mu$, there are more holes $\left(n_{h}\right)$ than electrons $\left(n_{e}\right)$ involved. It is of interest to see how large the asymmetry in $W_{C R}$ and $\omega_{\mathrm{CR}}$ are when plotted with respect to an equal value of doping (holes or electrons). To accomplish this conversion, we use the relationship between $\mu$ and $n$. Namely,

$$
\sqrt{\frac{4 \pi n}{\left(2 m v_{F}^{2}\right)^{2}}}=\left|1-\sqrt{1+\frac{2 \mu}{m v_{F}^{2}}}\right| .
$$


Using Eqn. (34), we find

$$
W_{C R}=e^{2} \frac{\sqrt{4 \pi n}}{16}\left(1 \pm \frac{\sqrt{4 \pi n}}{2 m v_{F}^{2}}\right),
$$

and

$$
\omega_{\mathrm{CR}}=\frac{e B \hbar v_{F}^{2}}{2|\mu|}\left(2 \pm \frac{\sqrt{4 \pi n}}{2 m v_{F}^{2}}\right)\left(1 \pm \frac{\sqrt{4 \pi n}}{2 m v_{F}^{2}}\right),
$$

for the optical spectral weight and frequency of the cyclotron resonance line, respectively. In both cases, \pm corresponds to electrons and holes, respectively. Here,

$$
|\mu|=\frac{m v_{F}^{2}}{2}\left|\left[1 \pm \frac{\sqrt{4 \pi n}}{2 m v_{F}^{2}}\right]^{2}-1\right| .
$$

The main frame of Fig. 6 shows the $\mu$ dependence of the density $n$ in units of $\hbar^{-2} v_{F}^{-2} \mathrm{meV}^{2}$ for the pure relativistic case (dash-dotted green) and TI with $\mu>0$ (solid red) and $\mu<0$ (dashed blue). The intersection of the curves with the dotted vertical line at $|\mu|=20 \mathrm{meV}$ (circles) emphasizes the different electron densities at fixed $\mu$. The horizontal dotted line gives the different chemical potentials (marked by a $x$ ) associated with a fixed particle number ( $n=200$ in our units). As expected, for fixed $\mu$, there are more holes than electrons. To get a constant number of dopants, the magnitude of the chemical potential needs to be considerably larger in the electron case than for holes. The impact of this conversion from chemical potential to dopant density is shown in Fig. 7 for the optical spectral weight [frame (a)] and cyclotron resonance frequency [frame (b)]. Large differences between electrons and holes are noted.

Next, we consider how the optical spectral weight under the intraband line $\left(W_{\text {intra }}\right)$ [i.e. the area under the first peak of Fig. $4(\mathrm{a})]$ and its position in energy $\left(\omega_{\text {intra }}\right)$ at finite $B$ approach the cyclotron resonance results as $B$ is decreased. This is shown in Fig. 8, In the top frame, we reproduce as the dotted red curve, the results previously obtained in Fig. 5 (solid red) for the spectral weight of the cyclotron resonance line $W_{\mathrm{CR}}$ in units of $e^{2} / \hbar \mathrm{meV}$ as a function of chemical potential for $\mu>0$. This curve is to be compared with the related results for the optical spectral weight $W_{\text {intra }}$ under the intraband line. The dashed blue curve is for $B=0.5 \mathrm{~T}$ while the solid black curve corresponds to $B=0.1 \mathrm{~T}$. As the magnetic field is reduced and $\mu$ is increased, our results for $W_{\text {intra }}$ approach the dotted red curve for $W_{\mathrm{CR}}$ as we expect. The two curves should agree in the limit of $\mu$ much larger than the magnetic energy. For $B=0.1 \mathrm{~T}$, this condition is $\mu \gg 3.3 \mathrm{meV}$. In Fig. 8 (b), we show the energy of the intraband transitions $\omega_{\text {intra }}$ in $\mathrm{meV}$ as a function of $\mu$ for the same two values of magnetic field: $B=0.5 \mathrm{~T}$ (dashed blue) and $B=0.1 \mathrm{~T}$ (solid black). The dotted green and red curves are for comparison and give $\omega_{\mathrm{CR}}$ vs. $\mu$ for $B=0.5 \mathrm{~T}$ and $0.1 \mathrm{~T}$, respectively. As the value of $\mu$
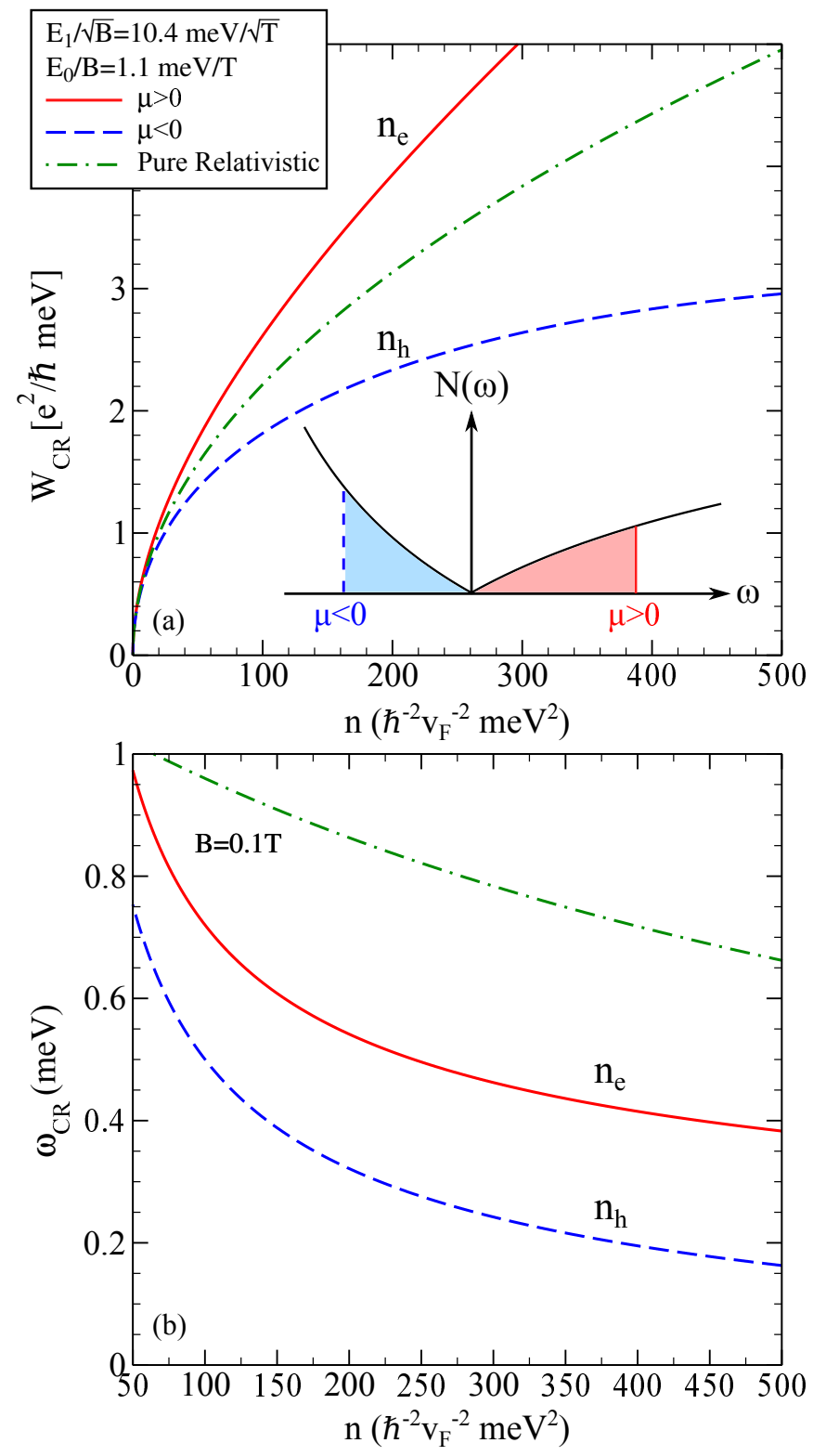

FIG. 7. (Color online) (a) Optical spectral weight under the cyclotron resonance line as a function of the number of dopants $n$ for the pure relativistic and TI regimes. Inset: density of states as a function of $\omega$. The valence and conduction portions are given by the blue and red shading, respectively. (b) The corresponding results for the cyclotron frequency at $B=0.1 \mathrm{~T}$.

is increased, the condition that $\mu$ be much greater than the magnetic energy scale is better satisfied and $\omega_{\text {intra }}$ merges with $\omega_{\mathrm{CR}}$.

\section{THE INTERBAND BACKGROUND}

In the previous section, we emphasized the intraband contribution to the optical conductivity. However, the 


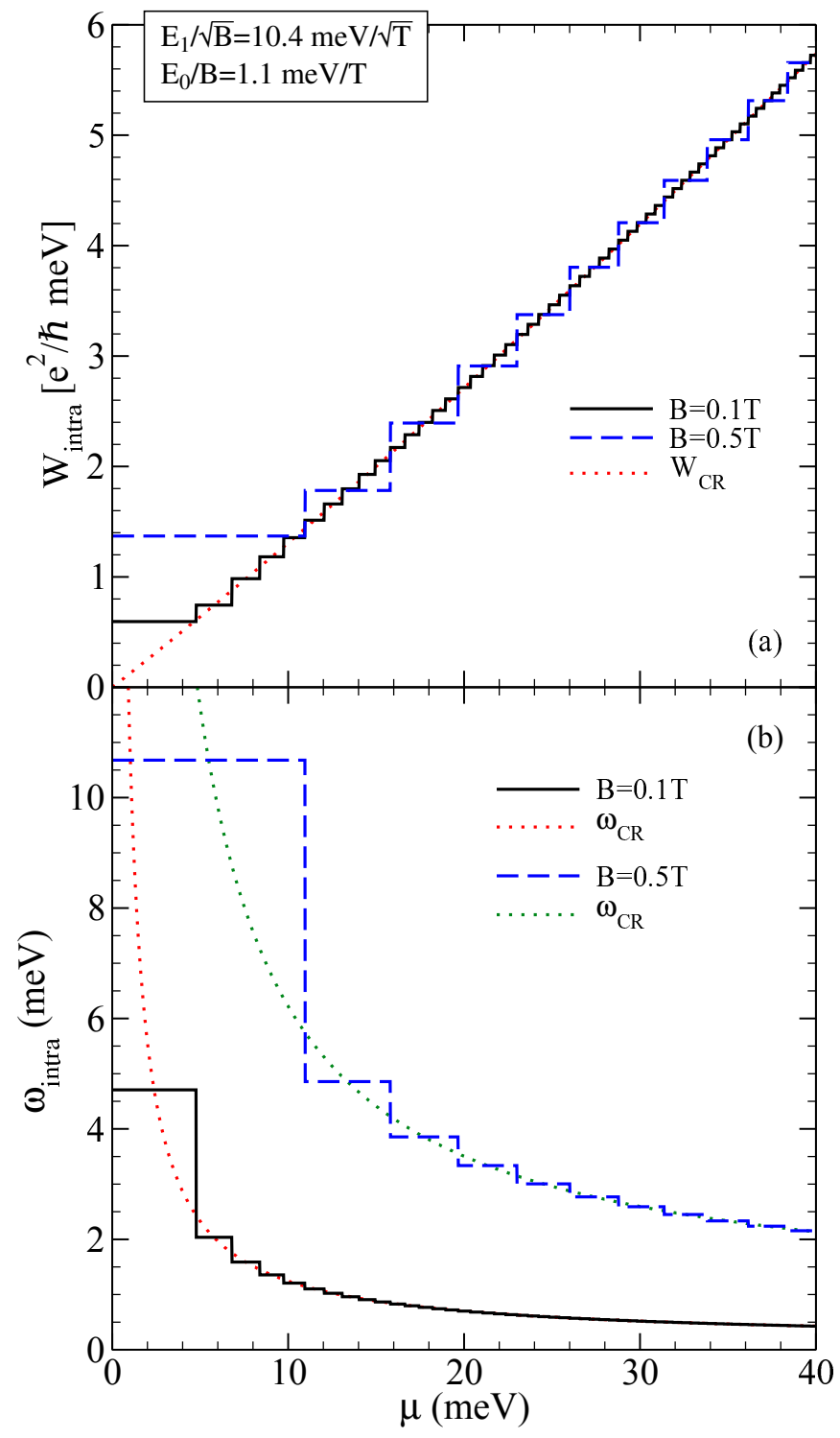

FIG. 8. (Color online) (a) Optical spectral weight under the intraband line as a function of $\mu$ for a TI. Two values of magnetic field are shown: $B=0.1 \mathrm{~T}$ and $B=0.5 \mathrm{~T}$. The dotted curves give the corresponding results for the cyclotron resonance spectral weight. (b) The position in energy of the intraband line for the same parameters as above.

interband part is also of interest and corresponds to all but the first magneto-optical lines of Figs. 2, 4, At larger values of photon energy and in the small $B$ limit, these provide the universal background which is given by the known formula

$$
\operatorname{Re} \sigma_{x x}(\Omega)=\frac{e^{2}}{16 \hbar} \Theta\left(\Omega-\max \left[2 \Delta, \omega_{\min }\right]\right),
$$

where $\omega_{\min }$ is the threshold energy for the onset of interband transitions. In Ref. $\underline{\underline{47}}$, this formula is derived from the form of the conductivity in the case of $B=0$ which involves an integration over momentum. Eqn. (38) prop- erly reduces to the graphene result $\underline{50,51}$ which differs from this equation only in that $\omega_{\min }=2 \mu$. In the appendix, we provide an alternative derivation of this formula. We start with Eqn. (12) for the longitudinal conductivity at finite $B$ and take the limit of $B \rightarrow 0$. This serves as a check on our work. The inset of Fig. 9 identifies the energy $\omega_{\min }$ for a particular value of $|\mu|$. It is smaller

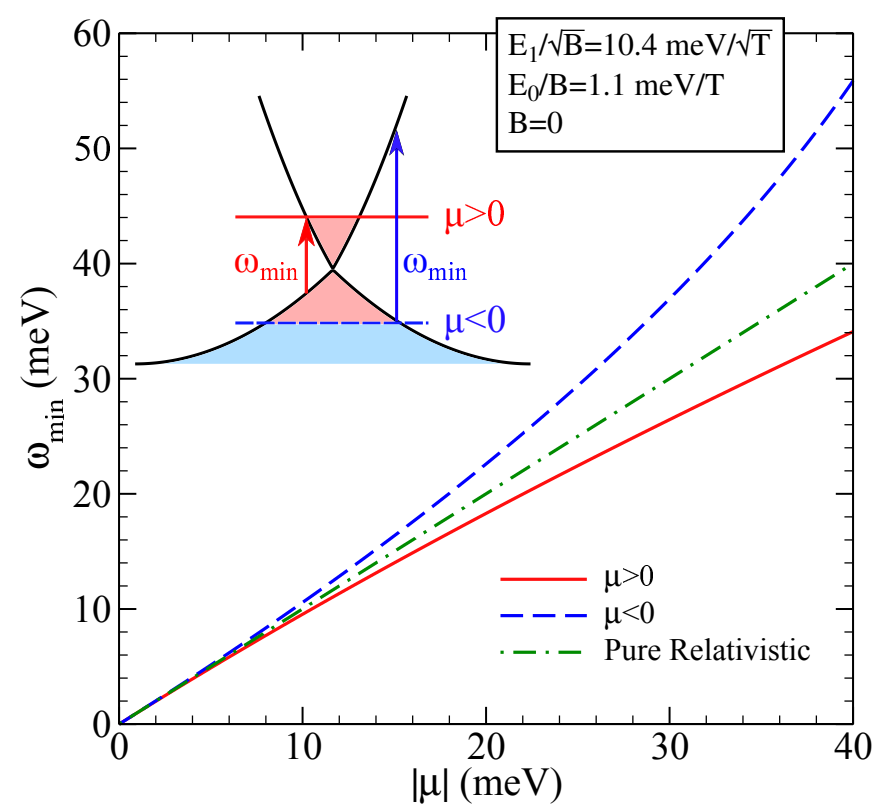

FIG. 9. (Color online) Energy cutoff of interband transitions as a function of $|\mu|$. Positive and negative $\mu$ are considered. The results are compared to the pure Dirac limit. Inset: sketch of the dispersion curves. The arrows identify the interband transitions that define $\omega_{\min }$. Blue corresponds to $\mu<0$ while red is for $\mu>0$.

for $\mu>0$ (red arrow) than for $\mu<0$ (blue arrow). The value of $\omega_{\text {min }}$ is determined by $\mathcal{E}_{+}\left(k_{c}\right)-\mathcal{E}_{-}\left(k_{c}\right)$ with $k_{c}$ corresponding to $\mu=\mathcal{E}_{+}\left(k_{c}\right)$ for $\mu>0$ and $\mu=\mathcal{E}_{-}\left(k_{c}\right)$ for $\mu<0$. Here, $\mathcal{E}_{ \pm}(k) \equiv \hbar^{2} k^{2} /(2 m) \pm \hbar v_{F} k$. This gives

$$
\omega_{\min }(\mu)=2 m v_{F}^{2}\left|1-\sqrt{1+\frac{2 \mu}{m v_{F}^{2}}}\right|
$$

which reduces to the known value of $2 \mu$ in the pure relativistic limit. For a TI with a subdominant nonrelativistic contribution, our results for $\omega_{\min }$ vs. $|\mu|$ are presented in the main frame of Fig. 9, The dash-dotted green curve is for comparison $(m \rightarrow \infty)$. For $\mu>0$, the curve (solid red) falls below the green line. For $\mu<0$, $\omega_{\min }$ (dashed blue) is above the relativistic limit. As we expect, all three curves come together at small $|\mu|$ since, in that limit, the relativistic linear-in-momentum magnetic energy dominates the non-relativistic quadratic term. As $|\mu|$ is increased, deviations from the pure relativistic result become significant.

In Fig. 10(a), we show both the intraband line (Drudelike peak near $\Omega=0$ ) and the constant interband background which sets in at $\Omega=\omega_{\min }$. The absolute value of 


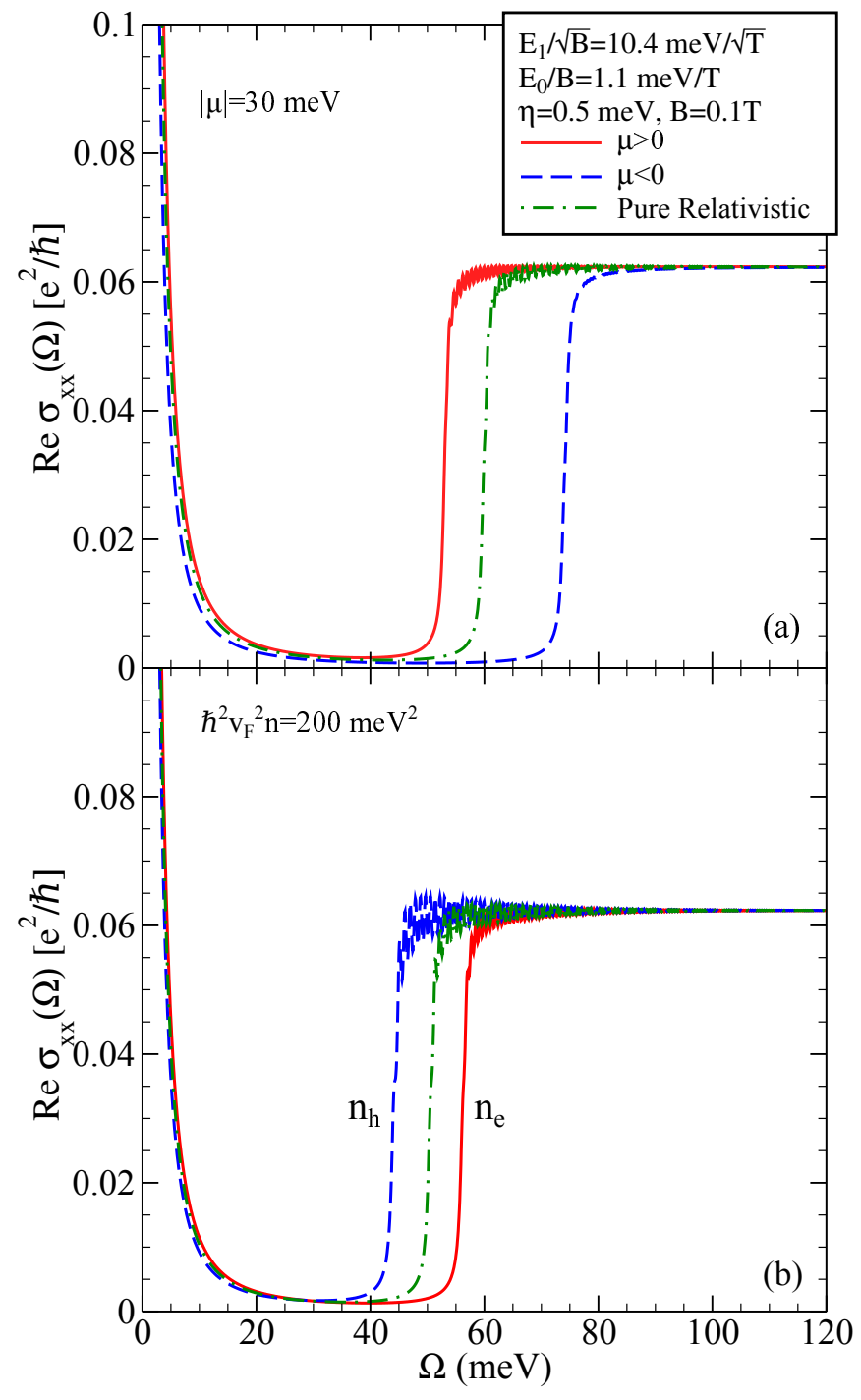

FIG. 10. (Color online) Real part of the longitudinal AC conductivity as a function of photon energy. (a) $|\mu|$ set at $30 \mathrm{meV}$. A different interband onset is seen for $\mu \lessgtr 0$ when the Schrödinger term is finite. (b) Corresponding results for a fixed dopant concentration $n \hbar^{2} v_{F}^{2}=200 \mathrm{meV}^{2}$.

$\mu$ is set at $30 \mathrm{meV}$ so that the various onsets for $\mu>0$ (solid red) and $\mu<0$ (dashed blue) in a TI, and the pure Dirac curve (dash-dotted green) are clearly separated. The height of the background remains at $(1 / 16) e^{2} / \hbar$ independent of the mass term. In frame (b), we show results for fixed dopant density $n$ set at $200 \hbar^{-2} v_{F}^{-2} \mathrm{meV}^{2}$. Again, the onset of interband transitions is changed by the subdominant Schrödinger term. Note that these results were obtained from finite magnetic field calculations for $B=0.1 \mathrm{~T}$ and consequently small wiggles from the underlying LL structure remain. These wiggles are most pronounced for small values of photon energy. Since we have included a residual scattering rate $\eta=0.5 \mathrm{meV}$, the Drude term centred at $\Omega=0$ is broadened. It would be a
Dirac $\delta$-function in the clean limit. This smearing parameter is also responsible for the small but finite conductivity seen between the Drude and interband contributions. Increasing the residual scattering rate would increase the value of this finite conductivity and reduce the small wiggles seen in the interband transitions of Fig. 10.

\section{DISCUSSION AND CONCLUSIONS}

We start with the Kubo formula for the real part of the longitudinal and transverse (Hall) conductivities in a magnetic field $B$ which is oriented perpendicular to the surface of a TI. The magnetic field creates LLs which lead to absorption peaks in the real part of the longitudinal optical conductivity. The introduction of a subdominant Schrödinger contribution to the Hamiltonian is known to split each interband peak into a doublet. Here, we find that there are associated peaks in the real part of the dynamical Hall conductivity $\operatorname{Re} \sigma_{x y}(\Omega)$ which are entirely due to the non-relativistic term.

A primary interest is the semiclassical regime which emerges when the magnitude of the chemical potential $|\mu|$ is much larger than both the relativistic $\left(E_{1}\right)$ and nonrelativistic $\left(E_{0}\right)$ magnetic energy scales. Nevertheless, we have found it illuminating to begin with a study of the evolution of both the dynamical conductivities $\operatorname{Re} \sigma_{x x}(\Omega)$ and $\operatorname{Re} \sigma_{x y}(\Omega)$ as the magnitude of the magnetic field is gradually reduced towards the zero-field limit. For $B=0$, the intraband optical transitions at finite doping provide a Drude contribution to $\operatorname{Re} \sigma_{x x}(\Omega)$ and the interband transitions give an additional universal background the height of which is independent of the Schrödinger mass; however, its onset is not. This step is also not particle-hole symmetric whether it be as function of $\mu$ or the number of dopants $(n)$. In the process of considering the interband background, we have provided a new derivation of the relevant formula. The derivation proceeds from our formula for $\operatorname{Re} \sigma_{x x}(\Omega)$ at finite magnetic field and the limit of $B \rightarrow 0$ is formally applied. At finite $B$, we find LL structures associated with the interband transitions in $\operatorname{Re} \sigma_{x y}(\Omega)$ which exist only when $m$ is finite. They are gradually reduced in amplitude with decreasing $B$; for $B=0$, the entire Hall conductivity is zero. For finite doping, the intraband transitions provide a peak in $\operatorname{Re} \sigma_{x x}(\Omega)$ at low photon energy $\Omega$ which evolves into the cyclotron resonance line as $B$ gets small or $|\mu|$ gets large (such that $|\mu|$ is much greater than $E_{1}$ and $E_{0}$ ). We find a corresponding peak-valley structure in $\operatorname{Re} \sigma_{x y}(\Omega)$ with its DC limit equal to the Hall plateau quantization which keeps the pure relativistic limit sequence of $( \pm 1 / 2, \pm 3 / 2, \pm 5 / 2, \ldots) e^{2} / h$. The value of $\mu$ at which the transition from one plateau to the next occurs is affected by the non-relativistic mass term. One consequence of this fact is that at charge neutrality $\left(\mu=0^{+}\right)$, the DC Hall conductivity (in units of $e^{2} / h$ ) becomes $-1 / 2$ for $E_{0} \neq 0$ while it is $1 / 2$ for $E_{0}=0$. This also implies that the structure seen in $\operatorname{Re} \sigma_{x y}(\Omega)$ at small $\Omega$ is switched 
from valley first to peak first.

When the chemical potential is large compared to the Dirac and Schrödinger magnetic scales, the intraband line moves toward the semiclassical cyclotron resonance line. We provide a simple analytic formula for the cyclotron resonance frequency, its optical spectral weight and its line shape which is found to be unaltered from the pure relativistic case. However, both the cyclotron frequency $\left(\omega_{\mathrm{CR}}\right)$ and optical spectral weight $\left(W_{C R}\right)$ are changed. In addition, due to the Schrödinger-mass induced particlehole asymmetry, both $\omega_{\mathrm{CR}}$ and $W_{C R}$ are different for positive and negative $\mu$. Differences are also evident for a fixed doping of electrons or holes. We provide a new derivation of the universal interband background of the longitudinal conductivity. The work is based on the Kubo formula at finite magnetic field and a formal limit of $B \rightarrow 0$. We recover the result obtained when no magnetic field is considered. The height of the background is completely independent of the Schrödinger mass $m$ but its onset at $\Omega=\omega_{\min }$ is not. This onset not only depends on $m$ but also depends on the sign of the chemical potential or (for a fixed value of dopant density) whether they are electrons or holes.

\section{Appendix}

In this appendix, we provide a derivation of the universal background formula [Eqn. (38)] as the $B \rightarrow 0$ limit of Eqn. (12). The two optical matrix elements of interest are for transitions from $(N-1,-)$ to $(N,+)$ and $(N,-)$ to $(N+1,+)$. In addition, these are only needed for large $N$ with $N=N_{c}$ given by $\mu=\mathcal{E}_{N_{c},+}$. For simplicity, we have assumed $\mu>0$. The negative $\mu$ case follows from similar considerations. We can take $\eta \rightarrow 0$ in Eqn. (12) to get

$$
\begin{aligned}
& \operatorname{Re}\left\{\frac{\sigma_{x x}(\Omega)}{e^{2} / \hbar}\right\}=\frac{E_{1}^{2}}{2} \sum_{N=0}^{\infty} \\
& \left\{\frac{f_{N+1,+}-f_{N,-}}{\mathcal{E}_{N,-}-\mathcal{E}_{N+1,+}} \mathcal{F}(N-; N+1+) \delta\left(\Omega+\mathcal{E}_{N+1,+}-\mathcal{E}_{N,-}\right)\right. \\
& \left.+\frac{f_{N-1,+}-f_{N,-}}{\mathcal{E}_{N,-}-\mathcal{E}_{N-1,+}} \mathcal{F}(N-; N-1+) \delta\left(\Omega+\mathcal{E}_{N-1,+}-\mathcal{E}_{N,-}\right)\right\} .
\end{aligned}
$$

In the limit $B \rightarrow 0$ and large $\mu$, the Dirac $\delta$-functions in Eqn. (A.1) restrict $N$ to values near $N_{c}$ where $\Omega=$ $2 \sqrt{2 N_{c}} E_{1}$ which also needs to be larger that $\omega_{\min }$. The $\mathcal{F}$ factors can be replaced by $\mathcal{F}\left(N_{c}-; N_{c}+\right)$ and the energy denominators by $-2 \sqrt{2 N_{c}} E_{1}$ since $E_{0}$ goes to zero as $B \rightarrow 0$. Finally, the thermal factor with a - needs to be replaced by 1 and the one with + by 0 . This allows only transitions with an energy larger than $\omega_{\min }$ or $N \geq N_{c}$. Thus, we have

$$
\begin{aligned}
\operatorname{Re}\left\{\frac{\sigma_{x x}(\Omega)}{e^{2} / \hbar}\right\}= & \frac{E_{1}^{2}}{2} \sum_{N=0}^{\infty} \frac{1}{\sqrt{2 N_{c}} E_{1}} \mathcal{F}\left(N_{c}-; N_{c}+\right) \\
& \times \delta\left(\Omega+\mathcal{E}_{N,+}-\mathcal{E}_{N,-}\right)
\end{aligned}
$$

with $\Omega>\omega_{\min }$. The sum over $N$ can be replaced by an integral over a continuous variable $N$. Integration is then preformed on the Dirac $\delta$-function which gives a factor of $\left.\left[(d / d N) 2 \sqrt{2 N} E_{1}\right]^{-1}\right|_{N_{c}}$. Therefore,

$$
\operatorname{Re}\left\{\frac{\sigma_{x x}(\Omega)}{e^{2} / \hbar}\right\}=\frac{E_{1}^{2}}{2} \frac{1}{\sqrt{2 N_{c}} E_{1}} \mathcal{F}\left(N_{c}-; N_{c}+\right) \frac{\sqrt{2 N_{c}}}{2 E_{1}} .
$$

But, $\mathcal{F}\left(N_{c}-; N_{c}+\right)=1 / 4$; so,

$$
\operatorname{Re} \sigma_{x x}(\Omega)=\frac{e^{2}}{16 \hbar}
$$

which is completely independent of the Schrödinger mass $m$. However, this constant background starts at $\Omega=$ $\omega_{\min }$ and so, as we have seen, this onset does depend on $m$ as emphasized in Eqn. (38).

\section{ACKNOWLEDGMENTS}

We thank E. J. Nicol for support of this project. This work has been supported by the Natural Sciences and Engineering Research Council of Canada and, in part, by the Canadian Institute for Advanced Research.
${ }^{1}$ C. L. Kane and E. J. Mele, Phys. Rev. Lett. 95, 226801 (2005).

2 M. Z. Hasan and C. L. Kane,
Rev. Mod. Phys. 82, $3045(2010)$.
${ }^{3}$ X.-L. Qi and S.-C. Zhang,

Rev. Mod. Phys. 83, 1057 (2011).

4 J. E. Moore, Nature 464, 194 (2010).

5 J. E. Moore and L. Balents, Phys. Rev. B 75, 121306(R) (2007). 
${ }^{6}$ L. Fu, C. L. Kane, and E. J. Mele, Phys. Rev. Lett. 98, 106803 (2007).

7 D. Hsieh, D. Qian, L. Wray, Y. Xia, Y. S. Hor, R. J. Cava, and M. Z. Hasan, Nature 452, 970 (2008).

${ }^{8} \mathrm{~L}$. Fu and C. L. Kane, Phys. Rev. Lett. 100, 096407 (2008).

9 H.-J. Zhang, C.-X. Liu, X.-L. Qi, X. Dai, Z. Fang, and S.-C. Zhang, Nature Phys. 5, 438 (2009).

10 Y. Ando, J. Phys. Soc. Jpn. 82, 102001 (2013).

11 D. Hsieh, Y. Xia, D. Qian, L. Wray, J. H. Dil, F. Meier, J. Osterwalder, L. Patthey, J. G. Checkelsky, N. P. Ong, A. V. Fedorov, H. Lin, A. Bansil, D. Grauer, Y. S. Hor, R. J. Cava, and M. Z. Hasan, Nature 460, 1101 (2009).

12 D. Hsieh, Y. Xia, L. Wray, D. Qian, A. Pal, J. H. Dil, J. Osterwalder, F. Meier, G. Bihlmayer, C. L. Kane, Y. S. Hor, R. J. Cava, and M. Z. Hasan, Science 323, 919 (2009).

13 A. K. Geim and K. S. Novoselov, Nature Materials 6, 183 (2007).

14 A. H. Castro Neto, F. Guinea, N. M. R. Peres, K. S. Novoselov, and A. K. Geim, Rev. Mod. Phys. 81, 109 (2009).

15 Z. Li and J. P. Carbotte, Phys. Rev. B 86, 205425 (2012).

16 L. Stille, C. J. Tabert, and E. J. Nicol, Phys. Rev. B 86, 195405 (2012)

17 C. J. Tabert and E. J. Nicol, Phys. Rev. Lett. 110, 197402 (2013)

18 C.-X. Liu, X.-L. Qi, H. Zhang, X. Dai, Z. Fang, and S.-C. Zhang, Phys. Rev. B 82, 045122 (2010).

19 Z. Li and J. P. Carbotte, Phys. Rev. B 88, 045414 (2013).

20 J. Fuchs, F. Piechon, M. Goerbig, and G. Montambaux, Eur. Phys. J. B 77, 351 (2010).

21 A. R. Wright and R. H. McKenzie, Phys. Rev. B 87, 085411 (2013).

22 M. O. Goerbig, G. Montambaux, and F. Piechon, Europhys. Lett. 105, 57005 (2014).

23 C. J. Tabert and J. P. Carbotte, J. Phys.: Condens. Matter 27, 015008 (2015).

24 C. J. Tabert and J. P. Carbotte, Phys. Rev. B 91, 235405 (2015).

25 A. A. Taskin and Y. Ando, Phys. Rev. B 84, 035301 (2011).

26 A. D. LaForge, A. Frenzel, B. C. Pursley, T. Lin, X. Liu, J. Shi, and D. N. Basov, Phys. Rev. B 81, 125120 (2010)

27 X. Xi, C. Ma, Z. Liu, Z. Chen, W. Ku, H. Berger, C. Martin, D. B. Tanner, and G. L. Carr, Phys. Rev. Lett. 111, 155701 (2013)

28 B. C. Chapler, K. W. Post, A. R. Richardella, J. S. Lee, J. Tao, N. Samarth, and D. N. Basov, Phys. Rev. B 89, 235308 (2014).

29 A. B. Sushkov, G. S. Jenkins, D. C. Schmadel, N. P. Butch, J. Paglione, and H. D. Drew, Phys. Rev. B 82, 125110 (2010).

30 G. S. Jenkins, D. C. Schmadel, A. B. Sushkov, H. D. Drew, M. Bichler, G. Koblmueller, M. Brahlek, N. Bansal, and S. Oh, Phys. Rev. B 87, 155126 (2013)

31 L. Wu, W.-K. Tse, M. Brahlek, C. M. Morris, R. Valdés Aguilar, N. Koirala, S. Oh, and N. P. Armitage, (2015),
arXiv:1502.04577

32 J. N. Hancock, J. L. M. van Mechelen, A. B. Kuzmenko, D. van der Marel, C. Brüne, E. G. Novik, G. V. Astakhov, H. Buhmann, and L. W. Molenkamp, Phys. Rev. Lett. 107, 136803 (2011).

33 C. Brüne, C. X. Liu, E. G. Novik, E. M. Hankiewicz, H. Buhmann, Y. L. Chen, X. L. Qi, Z. X. Shen, S. C. Zhang, and L. W. Molenkamp, Phys. Rev. Lett. 106, 126803 (2011).

34 A. A. Schafgans, K. W. Post, A. A. Taskin, Y. Ando, X.-L. Qi, B. C. Chapler, and D. N. Basov, Phys. Rev. B 85, 195440 (2012),

35 A. A. Taskin and Y. Ando, Phys. Rev. B 80, 085303 (2009).

36 R. Valdés Aguilar, J. Qi, M. Brahlek, N. Bansal, A. Azad, J. Bowlan, S. Oh, A. J. Taylor, R. P. Prasankumar, and D. A. Yarotski, Applied Physics Letters 106, 011901 (2015)

37 A. Crepaldi, F. Cilento, B. Ressel, C. Cacho, J. C. Johannsen, M. Zacchigna, H. Berger, P. Bugnon, C. Grazioli, I. C. E. Turcu, E. Springate, K. Kern, M. Grioni, and F. Parmigiani, Phys. Rev. B 88, 121404 (2013).

38 J. A. Sobota, S.-L. Yang, D. Leuenberger, A. F. Kemper, J. G. Analytis, I. R. Fisher, P. S. Kirchmann, T. P. Devereaux, and Z.-X. Shen, Phys. Rev. Lett. 113, 157401 (2014).

39 R. C. Hatch, M. Bianchi, D. Guan, S. Bao, J. Mi, B. B. Iversen, L. Nilsson, L. Hornekær, and P. Hofmann, Phys. Rev. B 83, 241303 (2011).

40 Z.-H. Pan, A. V. Fedorov, D. Gardner, Y. S. Lee, S. Chu, and T. Valla, Phys. Rev. Lett. 108, 187001 (2012)

41 T. Kondo, Y. Nakashima, Y. Ota, Y. Ishida, W. Malaeb, K. Okazaki, S. Shin, M. Kriener, S. Sasaki, K. Segawa, and Y. Ando, Phys. Rev. Lett. 110, 217601 (2013)

42 M. Brahlek, N. Koirala, M. Salehi, N. Bansal, and S. Oh, Phys. Rev. Lett. 113, 026801 (2014).

43 B. J. Lawson, G. Li, F. Yu, T. Asaba, C. Tinsman, T. Gao, W. Wang, Y. S. Hor, and L. Li, Phys. Rev. B 90, 195141 (2014).

44 G. Eguchi, K. Kuroda, K. Shirai, A. Kimura, and M. Shiraishi, Phys. Rev. B 90, 201307 (2014).

${ }^{45}$ K. Kuroda, M. Ye, A. Kimura, S. V. Eremeev, E. E. Krasovskii, E. V. Chulkov, Y. Ueda, K. Miyamoto, T. Okuda, K. Shimada, H. Namatame, and M. Taniguchi, Phys. Rev. Lett. 105, 146801 (2010)

46 Z. Li and J. P. Carbotte, Phys. Rev. B 89, 085413 (2014).

47 Z. Li and J. P. Carbotte, Phys. Rev. B 91, 115421 (2015)

48 Y. A. Bychkov and E. I. Rashba, J. Phys. C: Solid State Phys. 17, 6039 (1984).

49 Y. A. Bychkov and E. I. Rashba, JETP Lett. 39, 78 (1984).

50 V. P. Gusynin, S. G. Sharapov, and J. P. Carbotte, New J. Phys. 11, 095013 (2009).

51 V. P. Gusynin, S. G. Sharapov, and J. P. Carbotte, Phys. Rev. Lett. 96, 256802 (2006). 